\title{
A case of inclusion body myositis with benign monoclonal gammopathy successfully responding to repeated immunoabsorption
}

\author{
T Nakayama, E Horiuchi, T Watanabe, S Murayama, H Nakase
}

\begin{abstract}
A 69 year old woman with inclusion body myositis is described. She presented with benign monoclonal gammopathy. She was resistant to steroid therapy, but responded to repeated immunoabsorption. $U p$ to now, there has been no established therapy for inclusion body myositis, including IVIg. It is suggested that immunoabsorption could be an alternative therapy for inclusion body myositis, when it was accompanied by immunological abnormality.

(F Neurol Neurosurg Psychiatry 2000;68:230-233)
\end{abstract}

Keywords: inclusion body myositis, benign monoclonal gammopathy, steroid therapy, immunoabsorption, IgG

Inclusion body myositis is a common acquired myopathy in patients older than 50 . The characteristic pathological features are inflammation, vacuolated muscle fibres, amyloid deposits and $15-18 \mathrm{~nm}$ tubulo filaments. ${ }^{1}$ Mendell et al postulated the diagnostic criteria of inclusion body myositis. ${ }^{1}$ They diagnosed patients who exhibited all the above pathological features as definite inclusion body myositis and if the muscle only showed inflammation without the other pathological features, as possible inclusion body myositis. Dalakas et al reported that inclusion body myositis was often associated with monoclonal gammopathies. ${ }^{2}$ We examined a 69 year old woman with inclusion body myositis associated with IgG- $\gamma$, BJP- $\gamma$ type benign monoclonal gammopathy, which was successfully treated with repeated immunoabsorption.

\section{Case report}

A 69 year old woman developed muscle weakness from 66 years of age. There was no family history of neuromuscular disorders and no consanguinity. She was employed at a clothing shop as a deskworker. History disclosed dysaesthesia on the palmer side of her right second to fourth fingers at 67. She was
Department of

Neurology, Toranomon

Hospital, 2-2-2,

Toranomon,

Minato-ku, Tokyo

105-8470, Japan

T Nakayama

E Horiuchi

$\mathrm{T}$ Watanabe

H Nakase

Department of

Neurology, Division of

Neuroscience,

Graduate school of

Medicine, University

of Tokyo, 7-3-1, Hongo,

Bunkyo-ku, Tokyo

113-0033, Japan

S Murayama

Correspondence to:

Dr H Nakase,

Department of Neurology,

Toranomon Hospital, 2-2-2,

Toranomon, Minato-ku,

Tokyo 105-8470, Japan

email nakase-tky@umin.ac.jp

Received 5 March 1999 and in revised form

15 July 1999

Accepted 16 August 1999

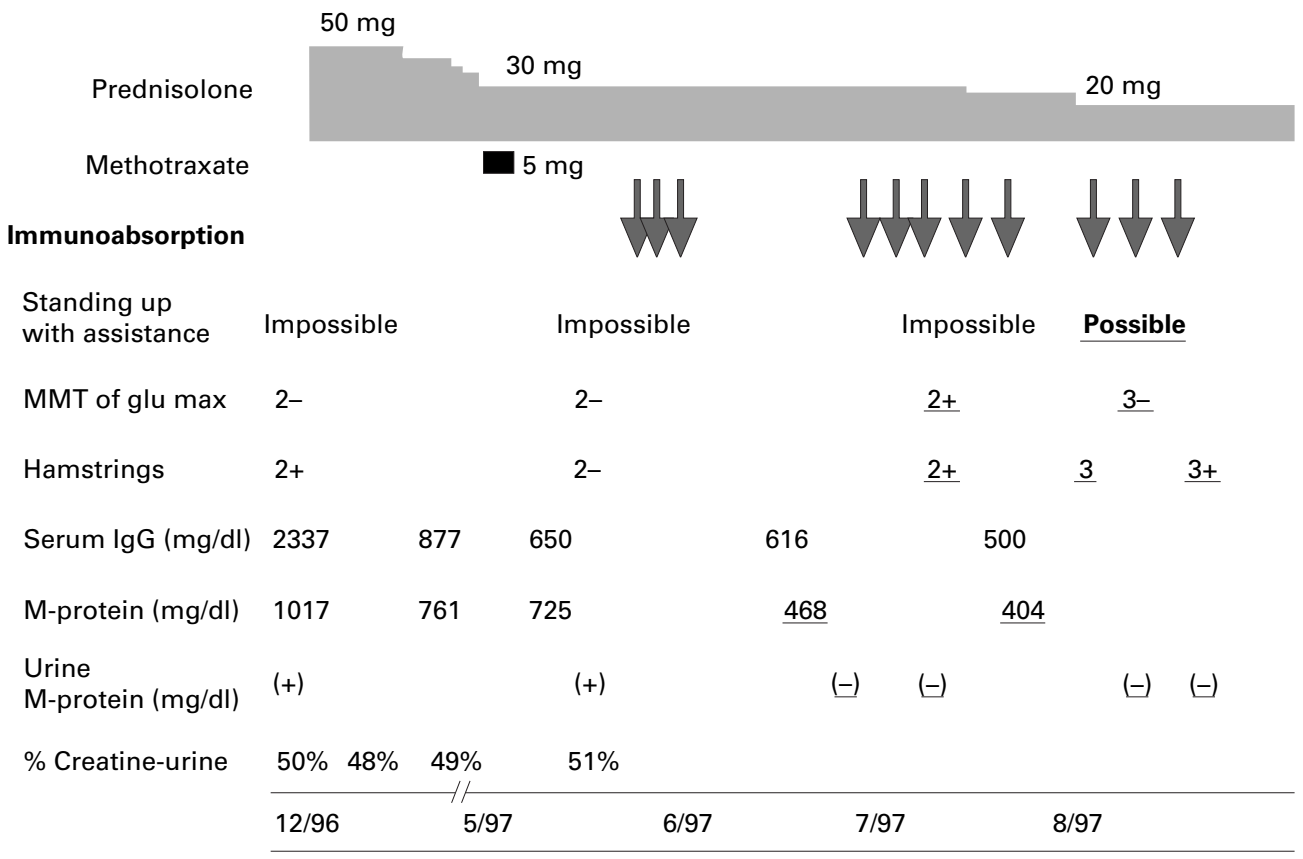

Figure 1 Clinical course of the patient. MMT=Manual muscle strength test; arrows indicate immunoabsorption treatments; clinical improvements are underlined. 

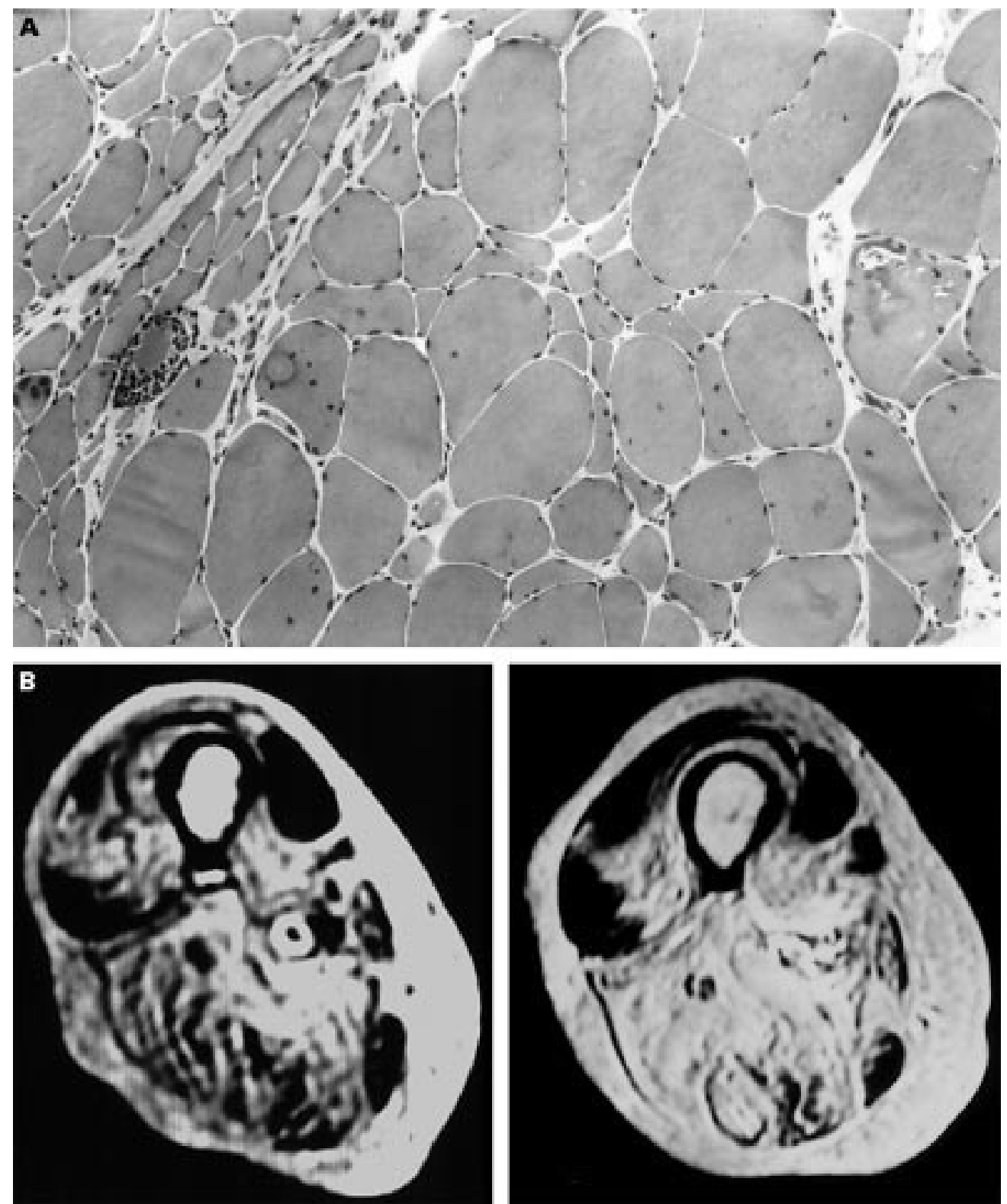

Figure 2 (A) Haematoxylin-eosin stain. (×33); Increase of connective tissue, variation in muscle fibre size, round muscle fibres in some groups, and increase of intermyonuclei are found. Some basophilic round nuclei in some regeneration fibres and rimmed vacuoles in a few muscle fibres are present. One basophilic fibre mononuclear infiltration is seen. (B) Muscle MRI of the right thigh muscle; T2 weighted images. The left image shows muscle atrophy in the thigh before prednisolone therapy. After repeated immunoabsorption, progression of muscle atrophy is not shown in the ight image.

diagnosed with carpal tunnel syndrome from the delayed distal latency of the right median nerve shown in a nerve conduction study. Endoscopic ligament resection was performed and her dysaesthesia disappeared.

At 66 years of age, she noticed weakness in her thigh muscles. At 67, she needed support standing up from a chair. At 68 , she went up stairs one step at a time and she walked more slowly than before. Later she could not go up stairs and needed help with housework. She could not stand up from a squat position, so she was admitted to our hospital in September 1996.

On admission, her weight had decreased by 6 $\mathrm{kg}$ over 3 years. Neurological examination showed atrophy of her hip and hamstring muscles, proximal dominant muscle weakness (manual muscle strength test gave the follow- ing results: neck flexion 3, extension 4 , arm 4 , forearm 4, hip extension 3-, hip flexion 2+, knee extension 2+, knee flexion 2-, ankle dorsiflexion 4, ankle planter flexion 5-), and dysaesthesia at the bilateral palmer site of the second to fourth fingers. Laboratory studies showed the following; creatine kinase and lactate dehydrogenase were moderately increased to 550IU/1 and 400IU/1, homogeneous type of antinuclear antibody was detected at 40 times dilution, IgG and BJP monoclonal gammaglobulins were detected. IgG was 650 $\mathrm{mg} / \mathrm{dl}$, IgA was $78.2 \mathrm{mg} / \mathrm{dl}$, and IgM was 114 $\mathrm{mg} / \mathrm{dl}$.. Urinalysis gave a \%creatine : creatinine ratio of $50 \%$. Bone marrow aspiration showed hypocellular bone marrow and its plasma cell concentration was $8.2 \%$. Bone radiography showed osteolytic lesions on the left temporal bone, the right fifth and sixth ribs and the left 
second and third ribs. Therefore, we concluded that her gammopathy was benign monoclonal gammopathy of uncertain significance (MGUS) from the Southwestern Oncology Group criteria for multiple myeloma.

Needle electromyography showed early recruitment of all examined muscles and at voluntary contraction polyphasic, low amplitude, and short duration unit potentials were revealed. At rest, a fibrillation and positive sharp wave was shown. We concluded that these were myogenic changes. Muscle CT showed severe atrophy of the bilateral gluteus maximum muscle and the hamstrings. Moderate atrophy of the paraspinal and bilateral posterior tibial muscles was also found.

Left semitendinosus muscle biopsy was performed. Haematoxylin-eosin staining showed increased connective tissue, variation in muscle fibre size, round muscle fibres in some groups, and increased intermyonuclei. We found basophilic round nuclei in some regenerating fibres; therefore, internuclear inclusions were suspected (fig $1 \mathrm{~A}$ ). Immunocytochemistry showed mononuclear infiltration around one basophilic fibre, prominent CD3 positive $\mathrm{T}$ cells, and dominant CD 8 positive cells. Gomori-trichrome stain showed rimmed vacuoles in a few muscle fibres. Tiny deposits of tau-positive profiles were detected. Characteristic filamentous inclusions were not disclosed by electron microscopy in the cytoplasm or myonuclei. We concluded that the patient had inclusion body myositis by the clinical criteria Mendel et al. ${ }^{2}$

Prednisolone $(50 \mathrm{mg} /$ day) was given from December 1996. Muscle power increased, but on tapering prednisolone the power decreased again. The $\%$ creatine : creatinine ratio of about $50 \%$ did not alter with the tapering of prednisolone. Steroid myopathy was excluded.

In April 1997, prednisolone was tapered to $30 \mathrm{mg} /$ day and methotraxate at $5 \mathrm{mg} /$ week was administered. Her hip flexor power was decreased to 2- and we concluded that immunosuppressive therapy with prednisolone and methotraxate had little effect.

Next we examined monoclonal gammopathy; we started immunoabsorption at the end of May 1997, 2-3 times a month. After the fourth absorption, monoclonal gammaglobulin in the urine disappeared and that in serum reduced. After the seventh absorption her knee flexion power was increased to 3-. After the ninth absorption, she could flex the knee at a right angle, and keep her leg up in a prone position; with assistance she could stand up from a chair, and her creatine kinase concentration reduced to $50 \mathrm{IU} / 1$. We tapered prednisolone gradually to $20 \mathrm{mg}$ /day from the the seventh absorption onwards and she showed no progression of muscle weakness. She was discharged from our hospital at the end of August 1997 and we continued absorption once a month, because monoclonal gammaglobulin in her urine reappeared 3 to 4 weeks after absorption. Her clinical course is shown below (fig 2) In April 1998, she had maintained the strength of her hamstring muscles. Muscle MRI of her thigh muscle showed muscle atrophy of the thigh similar to that before therapy; progression of muscle atrophy was not shown (fig $1 \mathrm{~B}$ ).

\section{Discussion}

Many studies have reported myopathy associated with MGUS or multiple myeloma Teleman-Toppet et al reported myositis with perifascicular atrophy associated with MGUS. ${ }^{3}$ Kiprov et al performed combination therapy of plasma exchange and treatment with prednisolone and cyclophosphamide for three cases of inflammatory myositis with perifascicular atrophy associated with IgG- $\gamma$ type MGUS. ${ }^{4}$ The treatment produced clinical improvement in all three patients. Examination of muscle biopsy specimens by direct immunofluorescence showed linear deposits of IgG- $\gamma$ along the sarcolemmal basement membrane. Muscle biopsy specimens after treatment showed no immune deposits. Sheehan-Dare and Simmons treated amyloid myopathy in association with IgG- $\gamma$ chain myeloma by melphalan and prednisolone $e^{5}$ which resulted in the remission of both the myeloma and myopathy. Eymard et al reported that plasmapheresis and immunosuppressive agents produced partial clinical improvement of late onset rod myopathy with MGUS, ${ }^{6}$ however, therapy interruption led to remission. As shown above, reduction of serum monoclonal gammaglobulin by chemotherapy, plasma exchange, or immunoabsorption improved the myositis.

Dalakas et al reported that inclusion body myositis was often associated with monoclonal gammaglobulin, ${ }^{2}$ but there was no report of plasma exchange or immunoabsorption to inclusion body myositis with gammopathy. Chad et al reported that plasma exchange produced no improvement of inclusion body myositis in Sjögren's syndrome ${ }^{7}$ but no control study was performed. Dalakas et al concluded that plasmapheresis was not helpful ${ }^{8}$ for inclusion body myositis because plasmapheresis was ineffective in a double blind controlled study conducted in polymyositis and dermatomyositis. Dalakas reported that IVIg treatment produced only modest improvement for inclusion body myositis ${ }^{9}$ and the efficacy of IVIg remains debatable. ${ }^{9} 10$

We examined inclusion body myositis with MGUS. Repeated immunoabsorption reduced urinary and serum monoclonal gammaglobulin and her muscle weakness was improved. The good response to immunoabsorption was similar to that of myositis with monoclonal gammaglobulin. It would be useful to look hard for immunological abnormalities such as MGUS or multiple myeloma in patients with inclusion body myositis and, if there, to consider immunoabsorption or plasmaphoresis.

1 Griggs RC, Askanas V, DiMauro S, et al. Inclusion myositis and myopathies. Ann Neurol 1995;38:705-13.

2 Dalakas MC, Illa I, Gallardo E, et al. Inclusion bosy myositis and paraproteinemia: incidence and immunopathologic correlations. Ann Neurol 1997;41:100-4.

3 Telerman-Toppet N, Wittek M, Bacq M, et al. Benign monoclonal gammopathy and relasping polymyositis. Muscle Nerve 1982;5:490-1.

4 Kiprov DD, Miller RG. Polymyositis associated with monoclonal gammopathy. Lancet 1984;ii:1183-6. 
5 Sheehan-Dare RA, Simmons AV. Amyloid myopathy and myeloma: response to treatment. Postgrad Med $\mathcal{F} 1987$, 63:141-2

6 Eymard B, Brouet JC, Collin H, et al. Late-onset rod myopathy associated with monoclonal gammopathy. Neu-

romusc Disord 1993;3:557-60.
7 Chad D, Good P, Adalman L, et al. Inclusion body myositis associated with Sjögren syndrome. Arch Neuro 1982;39:186.
8 Dalakas MC. Clinical, immunopathologic, and therpeutic considerations of inflamatory myopathies. Clin Neuropharmacol 1992;15:327-51.

9 Dalakas MC, Sonies B, Dambrosia J, et al. Treatment of inclusion-body myositis with IVIg: a double-blind, placebocontrolled study. Neurology 1997;48:712-16.

10 Barohn RJ. The therapeutic dilemma of inclusion body myositis. Neurology 1997;48:567-8. 